\title{
Single-Pole Interaction of the Particle with the String ${ }^{\star}$
}

\author{
Milovan VASILIĆ and Marko VOJINOVIĆ
}

Institute of Physics, P.O.Box 57, 11001 Belgrade, Serbia

E-mail:mvasilic@phy.bg.ac.yu,vmarko@phy.bg.ac.yu

Received October 24, 2007, in final form January 20, 2008; Published online February 12, 2008

Original article is available at http://www.emis.de/journals/SIGMA/2008/019/

\begin{abstract}
Within the framework of generalized Papapetrou method, we derive the effective equations of motion for a string with two particles attached to its ends, along with appropriate boundary conditions. The equations of motion are the usual Nambu-Goto-like equations, while boundary conditions turn out to be equations of motion for the particles at the string ends. Various properties of those equations are discussed, and a simple example is treated in detail, exhibiting the properties of Neumann and Dirichlet boundary conditions and giving a small correction term to the law of Regge trajectories due to the nonzero particle mass.
\end{abstract}

Key words: P-branes; classical theory of gravity; Regge trajectories; string theory

2000 Mathematics Subject Classification: 83C10; 83C55; 81T30; 70S10

\section{Introduction}

The interest in studying extended objects in high energy physics began with the observation that meson resonances could be viewed as rotating relativistic strings [6, 3]. This model provided a successful explanation of Regge trajectories. Nevertheless, the model assumes that the two quarks attached to the string have zero mass and zero angular momentum. The purpose of this short note is to take into account small mass of the two particles, and exhibit some basic properties of equations of motion in this case.

In order to do so, one needs to derive the equations of motion for the string with particles attached to its ends. The general method that lends itself for doing this was developed in [8], and represents the generalization of the Mathisson-Papapetrou method [5, 7] to include extended objects.

The layout of the paper is the following. In Section 2 we formulate the stress-energy tensor for the string with two particles attached in the simplest, single-pole approximation. Then we utilize its covariant conservation to derive equations of motion and boundary conditions for the string. It turns out that the boundary conditions are nothing but equations of motion for the particle residing at the end of the string. We recognize the force that acts upon the particle, and comment that in case of Nambu-Goto string this force has the form similar to Lorentz force from electrodynamics. We also comment on the particle mass conservation and the possibility of writing an action for the system of equations of motion.

In Section 3 we proceed to find an example solution of the equations of motion, where the string is of Nambu-Goto type and lying along a straight line of constant length $L$ while rotating with constant angular velocity about its center. The velocity of the particles at the ends turns out to depend on the length of the string, the mass of the particle and the string tension, in such a way that it is always less than the velocity of light, as expected. We discuss two limiting cases - in the limit of zero particle mass, the particle velocity becomes equal to the speed of

${ }^{\star}$ This paper is a contribution to the Proceedings of the Seventh International Conference "Symmetry in Nonlinear Mathematical Physics" (June 24-30, 2007, Kyiv, Ukraine). The full collection is available at http://www.emis.de/journals/SIGMA/symmetry2007.html 
light, and represents the situation analogous to imposing Neumann boundary conditions in usual string theory. In the limit of infinite particle mass, its velocity equals zero, and represents the situation analogous to imposing Dirichlet boundary conditions where the string is attached to a 0-brane, i.e. a particle. Other cases, those with finite particle velocity, do not appear in usual string theory.

Section 4 is devoted to computing the total energy and angular momentum for the example system discussed above. By eliminating the string length parameter from the equations, in the limit of small particle masses one recovers the familiar law of Regge trajectories, but now with a small correction term due to masses being small but still nonzero. This result is a demonstration how one can calculate corrections to the Regge law due to the presence of particles at the string ends.

In Section 5 we give our final comments and conclusions.

Our conventions are the same as in [8], aside from the metric signature, which we now take to be $\operatorname{diag}(-,+,+,+)$. Greek indices $\mu, \nu, \ldots$ are the spacetime indices, and run over $0,1, \ldots, D-1$. Latin indices $a, b, \ldots$ are the world sheet indices and run over $0,1, \ldots, p$. The Latin indices $i, j, \ldots$ refer to the world sheet boundary and take values $0,1, \ldots, p-1$. The coordinates of spacetime, world sheet and world sheet boundary are denoted by $x^{\mu}, \xi^{a}$ and $\lambda^{i}$, respectively. The corresponding metric tensors which are used to lower indices are denoted by $g_{\mu \nu}(x), \gamma_{a b}(\xi)$ and $h_{i j}(\lambda)$, while the indices are raised by the inverse metrics $g^{\mu \nu}, \gamma^{a b}$ and $h^{i j}$. We shall mainly be interested in the realistic case $D=4$.

\section{Equations of motion}

We begin by considering an isolated system consisted of a string with two massive particles attached to its ends, in Riemannian 4-dimensional spacetime. The basic starting point of the derivation of equations of motion is the covariant conservation of the symmetric stress-energy tensor $[7,8]$ :

$$
\nabla_{\nu} T^{\mu \nu}=0
$$

The stress-energy tensor is written as a sum of two terms,

$$
T^{\mu \nu}=\int_{\mathcal{M}} d^{2} \xi \sqrt{-\gamma} B_{\mathrm{s}}^{\mu \nu} \frac{\delta^{(4)}(x-z)}{\sqrt{-g}}+\int_{\partial \mathcal{M}} d \lambda \sqrt{-h} B_{\mathrm{p}}^{\mu \nu} \frac{\delta^{(4)}(x-z)}{\sqrt{-g}},
$$

representing the stress-energy components for the string and the particles at string endpoints, respectively. We restrict ourselves to the simplest, single-pole approximation, neglecting any higher terms containing derivatives of the Dirac delta function. The procedure described in [8] then yields the familiar world sheet equations

$$
\nabla_{a}\left(m^{a b} u_{b}^{\mu}\right)=0
$$

as well as expressions that determine the form of the stress-energy tensor:

$$
B_{\mathrm{s}}^{\mu \nu}=m^{a b} u_{a}^{\mu} u_{b}^{\nu}, \quad B_{\mathrm{p}}^{\mu \nu}=m v^{\mu} v^{\nu} .
$$

Here the world sheet is described by the parametrized equations $x^{\mu}=z^{\mu}\left(\xi^{a}\right)$, where $a \in\{0,1\}$. Similarly, the world sheet boundary is described by the parametrized equations $\xi^{a}=\zeta^{a}(\lambda)$ or equivalently $x^{\mu}=z^{\mu}\left(\zeta^{a}(\lambda)\right)$. Consequently, we define the notation for the vectors tangent to the world sheet and a vector tangent to the boundary:

$$
u_{a}^{\mu} \equiv \frac{\partial z^{\mu}}{\partial \xi^{a}}, \quad v^{a} \equiv \frac{\partial \zeta^{a}}{\partial \lambda}, \quad v^{\mu}=u_{a}^{\mu} v^{a} .
$$


Aside from equations of motion, one also gets the appropriate boundary conditions for the string. The particle part of $T^{\mu \nu}$ is constrained by the requirement that particle trajectories coincide with the string boundary, so the resulting boundary conditions are:

$$
\frac{D}{d s}\left(m v^{\mu}\right)=n_{a} m^{a b} u_{b}^{\mu} .
$$

These boundary conditions are written using the parametrization $\lambda=s$ (where $s$ is the proper distance parameter), or equivalently fixing the gauge condition $h \equiv v_{\mu} v^{\mu}=-1$, and are reinterpreted as the particle equations of motion. The stress-energy tensor of such a system has also been studied in [2].

The boundary conditions represent the equation determining particle trajectory, and the term on the right-hand side represents the force that string exerts on the particle. If the boundary $\partial \mathcal{M}$ consists of two disjoint lines, $\partial \mathcal{M}=\partial \mathcal{M}_{1} \cup \partial \mathcal{M}_{2}$ and $\partial \mathcal{M}_{1} \cap \partial \mathcal{M}_{2}=\varnothing$, the masses $m$ of the two particles may differ.

In general, equations (1) and (3) cannot be derived by extremizing some action if one does not introduce auxiliary variables in the theory. However, for the case of Nambu-Goto matter described by the choice

$$
m^{a b}=T \gamma^{a b}
$$

such an action exists, and is of the form

$$
S=T \int_{\mathcal{M}} d^{2} \xi \sqrt{-\gamma}-m \int_{\partial \mathcal{M}} d \lambda \sqrt{-h}
$$

The information that the particles are attached to string ends is encoded in the requirement that the domain of integration for the particle action be precisely the boundary of the string world sheet $\mathcal{M}$. As above, if the boundary consists of two disjoint lines, the particle part of the action can be split in two independent parts, each containing independent mass parameter, $m_{1}$ and $m_{2}$, which need not be equal. This action can be also used to model monopole-antimonopole pairs connected by Nambu-Goto strings, formed in phase transition in the early universe [1, 4].

There are two other peculiarities of the Nambu-Goto choice of matter, as we shall show. First, by contracting the equation (3) with $v_{\mu}$, and having in mind the choice $m^{a b}=T \gamma^{a b}$, one easily derives the law of mass conservation:

$$
\frac{d m}{d s}=0 .
$$

In the general case of $m^{a b}$ this need not hold, because matter may be allowed to flow from the particle to the interior of the string and vice versa.

Second, denoting the right-hand side of (3) as the force $F^{\mu}$, we can rewrite it as:

$$
F^{\mu} \equiv T n^{\mu}=T F^{\mu}{ }_{\nu} v^{\nu}, \quad F^{\mu \nu} \equiv u_{a}^{\mu} e^{a b} u_{b}^{\nu} .
$$

Here $e^{a b}$ is the covariant totally antisymmetric Levi-Civita tensor. In this notation, the equation of motion for the particle takes the form:

$$
m \frac{D}{d s} v^{\mu}=T F^{\mu \nu} v_{\nu}
$$

which looks identically the same as the well known Lorentz force law from ordinary electrodynamics:

$$
m \frac{D}{d s} v^{\mu}=q \mathcal{F}^{\mu \nu} v_{\nu}
$$

$\mathcal{F}^{\mu \nu}$ being the antisymmetric electromagnetic field strength tensor, and $q$ the appropriate charge of the particle. While the "string field strength tensor" $F^{\mu \nu}$ is also antisymmetric, it is of course of entirely different nature, but the identical form of the force law nevertheless seems interesting. 


\section{Neumann and Dirichlet boundary conditions}

In what follows, we shall assume that the string is made of the Nambu-Goto type of matter, moving in a 4-dimensional flat spacetime:

$$
m^{a b}=T \gamma^{a b}, \quad R_{\nu \lambda \rho}^{\mu}=0 .
$$

Then, the world sheet equations (1) reduce to the familiar Nambu-Goto equations, and the third term on the right-hand side of (3) becomes $T n^{\mu}$.

Now, we look for a simple, straight line solution of the equations of motion (1). This has also been discussed in [4], but for different purpose. Without loss of generality, we put

$$
\vec{z}=\vec{\alpha}(\tau) \sigma, \quad z^{0}=\tau,
$$

where $\xi^{0} \equiv \tau$ and $\xi^{1} \equiv \sigma$ take values in the intervals $(-\infty, \infty)$ and $[-1,1]$, respectively. This choice represents a string lying along the line of length $L$, in appropriate coordinates, with its center point at rest in coordinate origin. Assuming that the string length $L=2|\vec{\alpha}|$, and the velocity of the string ends $V=|d \vec{\alpha} / d \tau|$ are constant, the equation (1) reduces to

$$
\frac{d^{2}}{d \tau^{2}} \vec{\alpha}+\omega^{2} \vec{\alpha}=0, \quad \omega \equiv \frac{2 V}{L} .
$$

It describes uniform rotation in a plane. Choosing the rotation plane to be the $x-y$ plane, we get the solution

$$
\vec{\alpha}=\frac{L}{2}\left(\cos \omega \tau \vec{e}_{x}+\sin \omega \tau \vec{e}_{y}\right) .
$$

Next we consider the boundary equation (3). Omitting the details of the calculation, we find that the particle velocity becomes

$$
V=\frac{1}{\sqrt{1+\frac{2 m}{T L}}} .
$$

Of course, our assumption that the velocities of two ends are equal implies that in this case masses of two particles must be equal.

By inspecting the expression (4), we see that $V<1$, as it should be. In the limit $m \rightarrow 0$, the string ends move with the speed of light, representing the Nambu-Goto dynamics with Neumann boundary conditions, i.e. a free string. When $m \rightarrow \infty$, the string ends do not move. This is an example of Dirichlet boundary conditions, where string ends are attached to a fixed $p$-brane, in our case $p$ being equal to zero, i.e. the particle. All other choices for $m$ represent cases outside these two, and do not appear in ordinary string theory.

\section{Regge trajectories law}

The total angular momentum and energy of the example system considered in previous section are calculated using the usual definitions:

$$
E=\int d^{3} x T^{00}, \quad J^{\mu \nu}=\int d^{3} x x^{[\mu} T^{\nu] 0} .
$$

Substituting the appropriate solution into the expressions (2) and back into the stress-energy tensor, after integration one easily finds

$$
E=T L \frac{\arcsin V}{V}+\frac{2 m}{\sqrt{1-V^{2}}}, \quad J=\frac{T L^{2}}{4}\left(\frac{\arcsin V}{V^{2}}-\frac{\sqrt{1-V^{2}}}{V}\right)+\frac{2 m}{\sqrt{1-V^{2}}} \frac{L V}{2} .
$$


These equations have obvious interpretation. The total energy of the system consists of the string energy and the kinetic energy of the two particles, while the total angular momentum is the sum of the string orbital angular momentum and orbital angular momenta of the two particles.

In the limit of small particle masses, the free parameter $L$ can be eliminated in favour of $E$, which leads to

$$
J=\frac{E^{2}}{2 \pi T}-\frac{4}{3 T} \sqrt{\frac{E}{\pi}} m^{\frac{3}{2}}+\mathcal{O}\left(m^{2}\right) .
$$

The first term on the right-hand side defines the known Regge trajectory, while the second represents a small correction due to the presence of massive particles at the string ends. As we can see, the unique Regge trajectory of the ordinary string theory splits into a family of distinctive trajectories, and that $J$ becomes nonlinear in $E^{2}$.

Of course, this result is specific to the particular example discussed above, but it shows that one can in principle calculate corrections to the law of Regge trajectories by allowing the end-point particles to have nonzero mass.

\section{Concluding remarks}

In this paper we have analyzed the system consisting of a string with two particles attached to its ends. The method we use is a generalization of the Mathisson-Papapetrou method for pointlike matter [5, 7]. It has already been used in [8] for the derivation of equations of motion of extended objects. Using those results, we have derived the equations of motion for the string along with the appropriate boundary conditions. These boundary conditions turn out to be the equations of motion for the two particles attached to the string ends.

These equations of motion display the string force acting on the particle. In some cases they imply that the mass of the particle is conserved, and the force term can be rewritten in the form that is formally identical to Lorentz force law of electrodynamics. Also, in general case the equations of motion for the string and the particle do not allow themselves to be derived by extremizing some action, without introduction of auxiliary variables. However, in the special case of Nambu-Goto matter for the string, such an action does exist.

Next we specialized to the case of the usual Nambu-Goto string with two massive particles at its ends. The equations of motion can be solved exactly for the case of a straight line string rotating around its center. It turns out that the velocity of the string ends is less than the velocity of light, and is dependent on the masses of the particles. In this way, one is provided a way to describe both Neumann and Dirichlet boundary conditions for the Nambu-Goto string in the limits where the masses of the two particles approach zero or infinity, respectively.

Finally, given this solution, one can calculate the total energy and angular momentum of the system, and in the limit of small particle masses derive the relation connecting the total angular momentum with the total energy of the string and the particles. This relation represents the law of Regge trajectories, with a correction term due to the particle masses. In this setting, there is not only one Regge trajectory, but a whole family, due to arbitrariness in choice of mass parameters for the constituent particles. Also, the Regge trajectory ceases to be linear in $E^{2}$ in the limit of small but nonzero particle masses.

We remark at the end that more general configurations can also be treated using this formalism. It is an open question, however, whether the equations of motion of those configurations are integrable for some special case of motion. Also, the whole treatment presented in this paper is entirely classical, and one could in principle study the quantum theory of strings with particles attached to its ends. The spectra of perturbations of such strings is also an open question. Both these questions represent possible topics for future research. 


\section{Acknowledgments}

This work was supported by the Serbian Science Foundation, Serbia. A CEI grant for participation in the Seventh International Conference "Symmetry in Nonlinear Mathematical Physics" is gratefully acknowledged.

\section{References}

[1] Berezinsky V., Martin X., Vilenkin A., High energy particles from monopoles connected by strings, Phys. Rev. D 56 (1997), 2024-2034, astro-ph/9703077.

[2] Carter B., Mechanics of cosmic rings, Phys. Lett. B 238 (1990), 166-171.

[3] Goto T., Relativistic quantum mechanics of one-dimensional mechanical continuum and subsidiary condition of dual resonance model, Prog. Theoret. Phys. 46 (1971), 1560-1569.

[4] Martin X., Vilenkin A., Gravitational radiation from monopoles connected by strings, Phys. Rev. D 55 (1997), 6054-6060.

[5] Mathisson M., Neue Mechanik materieller Systeme, Acta Phys. Polon. 6 (1937), 163-200.

[6] Nambu Y., Strings, monopoles, and gauge fields, Phys. Rev. D 10 (1974), 4262-4268.

[7] Papapetrou A., Spinning test-particles in general relativity. I, Proc. R. Soc. Lond. Ser. A 209 (1951), $248-258$.

[8] Vasilić M., Vojinović M., Classical string in curved backgrounds, Phys. Rev. D 73 (2006), 124013, 12 pages, gr-qc/0610014. 